\title{
Hypoxic exosomes facilitate angiogenesis and metastasis in esophageal squamous cell carcinoma through altering the phenotype and transcriptome of endothelial cells
}

Yu Mao ${ }^{1 *}$, Yimin Wang ${ }^{2}$, Lixin Dong ${ }^{1}$, Yunjie Zhang ${ }^{1}$, Yanqiu Zhang ${ }^{1}$, Chao Wang ${ }^{3}$, Qiang Zhang ${ }^{1}$, Sen Yang ${ }^{1}$, Liyan Cao ${ }^{1}$, Xinyuan Zhang ${ }^{1}$, Xin $\mathrm{Li}^{1}$ and Zhanzhao $\mathrm{Fu}^{1 *}$

\begin{abstract}
Background: In cancer progression, hypoxia, or low oxygen tension, is a major regulator of tumor aggressiveness and metastasis. However, how cancer cells adapt to the hypoxia and communicate with other mesenchymal cells in microenvironment during tumor development remains to be elucidated. Here, we investigated the involvement of exosomes in modulating angiogenesis and enhancing metastasis in esophageal squamous cell carcinoma (ESCC).

Methods: Differential centrifugation, transmission electron microscopy and nanoparticle tracking analysis were used to isolate and characterize exosomes. Colony formation and transwell assay were performed to assess the proliferation, migration and invasion of human umbilical vein endothelial cells (HUVECs). The tube formation assay and matrigel plug assay were used to evaluate the vascular formation ability of HUVECs in vitro and in vivo respectively. An in vivo nude mice model was established to detect the regulatory role of exosomes in ESCC progression. Microarray analysis was performed to analyze the transcriptome profiles in HUVECs.

Results: Exosomes derived from ESCC cells cultured under hypoxia played a better role in promoting proliferation, migration, invasion and tube formation of HUVECs in vitro and in vivo than exosomes from ESCC cells cultured under normoxia. Moreover, hypoxic exosomes significantly enhanced the tumor growth and lung metastasis compared with normoxic exosomes in nude mice models. Interestingly, endothelial cells were programmed by hypoxic and normoxic exosomes from ESCC cells which altered the transcriptome profile of HUVECs.
\end{abstract}

Conclusions: Taken together, our data identified an angiogenic role of exosomes from ESCC cells which shed light on the further application of exosomes as valuable therapeutic target for ESCC.

Keywords: ESCC, Angiogensis, Exosomes, Metastasis

\footnotetext{
*Correspondence: ayabluewin@163.com; doctors12email@163.com

'Department of Oncology, First Hospital of Qinhuangdao, Wenhua Road No.

258, Haigang District, Qinhuangdao 066000, Hebei, China

Full list of author information is available at the end of the article
}

(c) The Author(s). 2019 Open Access This article is distributed under the terms of the Creative Commons Attribution 4.0 International License (http://creativecommons.org/licenses/by/4.0/), which permits unrestricted use, distribution, and reproduction in any medium, provided you give appropriate credit to the original author(s) and the source, provide a link to the Creative Commons license, and indicate if changes were made. The Creative Commons Public Domain Dedication waiver (http://creativecommons.org/publicdomain/zero/1.0/) applies to the data made available in this article, unless otherwise stated. 


\section{Background}

Esophageal cancer is one of the most common malignant tumors worldwide which seriously threatens human health [1]. Esophageal squamous cell carcinoma (ESCC) is the predominant form of esophageal cancer worldwide [2]. Conventional therapeutic strategies such as radiotherapy, chemotherapy and surgery show limited effect for ESCC treatment, especially for advanced ESCC with metastasis [3]. During cancer progression, tumors cannot grow beyond $1-2 \mathrm{~mm}$ without a vascular supply, due to an insufficient supply of oxygen, hypoxia, appears [4, 5]. Hypoxia is a major driver of tumor angiogenesis [5, 6]. As a multi-step physiological process, angiogenesis comprises several sequential steps involving the proliferation, migration, and morphogenesis of endothelial cells $[4,7,8]$. In tumor microenvironment (TEM), such coordination is partially accomplished by the transfer of angiogenic factors from tumor cells to endothelial cells $[4,9]$.

Recent studies has verified the potential role of exosomes as important signaling entities in the cross-talk between various cell types. Exosomes are vesicles of endocytic origin released by many cells $[10,11]$. The involvement of exosomes in esophageal cancer proliferation, metastasis, and drug resistance is becoming increasingly apparent [12-18]. Besides, exosomes can reflect the hypoxic status of cancer cells and further help them adapt to hypoxia through communicating with their surrounding microenvironment during tumor development [19]. However, whether exosomes play a role in modulating angiogenesis and, hence, helping the ESCC cells conquer the hypoxic microenvironment remains to be elucidated.

In the present study, the potential effect of exosomes from ESCC on endothelial cells in TEM was firstly explored. Moreover, by culturing the ESCC in hypoxic condition, we demonstrated that hypoxia might alter the content of exosomes secreted by ESCC cells and further modulate the activation of endothelial cells which internalized these exosomes.

\section{Material and methods}

\section{Cell lines and cell culture}

In order to obtain the exosomes-depleted fetal bovine serum (FBS), FBS was firstly centrifuged at $100,000 \times \mathrm{g}$ for $12 \mathrm{~h}$ in an ultracentrifuge (Beckman Coulter, Optima L-100XP, USA) at $4{ }^{\circ} \mathrm{C}$ and then discarded the pellet.

ECA109, KYSE410 and HET-1A cell lines were purchased from American Type Culture Collection. HET1A cell line was cultured in Epithelial Cell Growth Basal Medium (Lonza, Switzerland). ECA109 and KYSE410 cell lines were cultured in RPMI 1640 (Life Technologies/Invitrogen) supplemented with 10\% FBS (Gibco, New Zealand) and $1 \%$ penicillin/streptomycin (Gibco, New Zealand).
Primary human umbilical vein endothelial cells (HUVECs) were also purchased from American Type Culture Collection and maintained in endothelial cell medium (ECM) (Science cell, USA). All cells were cultivated in a humidified incubator at $37{ }^{\circ} \mathrm{C}$ with $5 \%$ $\mathrm{CO}_{2}$. For hypoxia experiments, cells were incubated in a humidified $5 \% \mathrm{CO}_{2}$ and $0.5 \% \mathrm{O}_{2}$ for 5 days at $37^{\circ} \mathrm{C}$.

\section{Exosomes isolation}

Exosomes were extracted from ESCC cell culture medium using differential centrifugation. To this end, culture medium $(9 \mathrm{ml})$ were collected from ESCC cells $\left(1 \times 10^{7}\right)$ cultured at normoxic or hypoxic conditions in a $10 \mathrm{~cm}$ diameter petri dish. Culture medium were centrifuged at $300 \mathrm{~g}-5 \mathrm{~min}$ to eliminate cell debris. Supernatant were further centrifuged at 16,500 g-30 min and 100,000 g-2 h. Finally, the exosome pellets were washed once in a large volume of phosphate buffer saline (PBS) and then followed by centrifugation (100,000 g-2 h). The exosomes were quantified by measuring the exosomal protein with $\mathrm{BCA}^{\mathrm{sm}}$ Protein Assay Kit (Pierce, USA).

\section{Transmission electron microscopy}

The exosome-enriched suspension were suspended in $50 \mu \mathrm{L}$ of PBS, fixed with paraformaldehyde and glutaraldehyde. Exosome sample was adsorbed onto a carboncoated copper grid and immersed in phosphotungstic acid solution for $30 \mathrm{~s}$. Then the samples were observed in a Zeiss transmission electron microscope (Zeiss, Germany).

\section{Nanoparticle tracking analysis}

The number of exosomes and the size distribution were analyzed using the Nanosight (Malvern, UK) and NTA analytical software (version 2.3, Nanosight).

\section{Western blotting analysis}

The purified exosomes and ECA109 or KYSE410 cells were lysed using RIPA buffer (Roche). The protein concentration of lysates was quantified with $\mathrm{BCA}^{\mathrm{rm}}$ Protein Assay Kit (Pierce, USA). Then proteins were separated by SDS-PAGE and transferred onto a PVDF membrane. The PVDF membrane was blocked with 5\% non-fat milk and incubated with the primary antibodies (CD9 and TSG101) (Cell Signaling Technology, USA) overnight at $4{ }^{\circ} \mathrm{C}$. The bands were probed with secondary antibody (Icllab, USA) and visualized by chemiluminescence (Millipore, MA, USA). The intensity of the protein bands was quantified by densitometry using Image J Software (National Institutes of Health). Each assay was repeated at least three times. One representative of three independent experiments was shown. 


\section{Colony formation assay}

As we previously depicted, HUVECs were seeded into 6well plates (500 cells/well) and incubated with medium containing exosomes $(25 \mu \mathrm{g} / \mathrm{mL})$ or not for 2 weeks. The colonies were stained with crystal violet for $15 \mathrm{~min}$ and then counted [20,21]. Each assay was repeated at least three times. One representative of three independent experiments was shown.

\section{Cell cycle analysis}

As we have previously depicted, HUVECs were fixed with ice-cold ethanol for $24 \mathrm{~h}$ and then dyed with propidium iodide/RNase buffer (BD Biosciences, USA) for 30 min in a dark place. Samples were analyzed by BD FACS Calibur flow cytometer. Data was analyzed using Modfit [20]. Each assay was repeated at least three times. One representative of three independent experiments was shown.

\section{Invasion and migration assay}

As we previously depicted, $0.1 \mathrm{~mL}$ FBS-free ECM containing $2 \times 10^{4}$ HUVECs, in the presence of exosomes $(25 \mu \mathrm{g} / \mathrm{mL})$ or not, were seeded into the upper chamber of transwell chambers (24 wells) (Merck, Germany) coated with or without Matrigel (BD Biosciences, USA). $0.6 \mathrm{~mL}$ of the ECM containing 10\% FBS was placed into the lower chamber. $48 \mathrm{~h}$ later, the bottom surface of the membranes were stained with crystal violet and photographed. The number of invasive cells were counted in three random microscopic fields under a light microscope [22]. Each assay was repeated at least three times. One representative of three independent experiments was shown.

\section{Exosomes labeling, internalization and confocal microscopy}

Exosomes were labeled with PKH26 Red Fluorescent marker (PKH26GL, Sigma-Aldrich, Germany) as recommended by the manufacturer. HUVECs were incubated with labeled exosomes $(25 \mu \mathrm{g} / \mathrm{mL})$ for varying times. Then HUVECs were fixed and stained with PhalloidiniFluor 488 Reagent (Abcam, UK) and DAPI (Solarbio, USA) according to the manufacturer's instruction. The images were acquired with a Zeiss Laser Scanning Confocal Microscope (Zeiss, Germany).

\section{Matrigel tube formation assay}

HUVECs were seeded into a Matrigel Basement Membrane Matrix (BD, New Jersey, USA) precoated 96well plate at $2 \times 10^{4}$ cells per well and cultured in ECM, in the presence of exosomes $(25 \mu \mathrm{g} / \mathrm{mL})$ or not. Calcein-AM (Sigma-Aldrich, Germany) was used to stain the HUVECs after seeding for $8 \mathrm{~h}$. The tube-like structures were imaged using the Zeiss fluorescence inverted microscope (Zeiss, Germany). The angiogenic property was assessed by measuring the total branching length from three random microscopic fields using Image J software (National Institutes of Health). Each assay was repeated at least three times. One representative of three independent experiments was shown.

\section{In vivo matrigel plug assay}

Five hundred ul of Matrigel (BD Biosciences) mixed with $(25 \mu \mathrm{g} / \mathrm{mL})$ normoxic or hypoxic exosomes was injected subcutaneously into the ventral region of BALB/c nude mice (Institute of Laboratory Animal Sciences, Chinese Academy of Medical Sciences (CAMS)). After inoculation for 7 days, the matrigel was excised and then fixed with formalin overnight, embedded in paraffin, and sectioned into slides. The plugs were stained with hematoxylin and eosin (H\&E) and visualized using the Zeiss inverted microscope (Zeiss, Germany) [23]. Three mice were used for each group. One representative of three independent experiments was shown.

\section{Expression profile analysis of RNAs in exosomes} HUVECs were divided into three groups: HUVECs incubated without exosomes (control group), HUVECs incubated with exosomes $(25 \mu \mathrm{g} / \mathrm{mL})$ from KYSE410 which cultured in hypoxic environment (hypo-Exo group) and HUVECs incubated with exosomes (25 $\mu \mathrm{g}$ $/ \mathrm{mL}$ ) from KYSE410 which cultured in normoxic environment (norm-Exo group). After incubation for 12 $\mathrm{h}$, the whole RNA of HUVECs was extracted using trizol (Thermo, USA). Gene expression profiling was performed by Beijing CNKINGBIO Biotechnology Company Limited using Clariom $^{\text {ma }}$ D Pico Assay, human (Affymetrix, USA) according to user guide.

Quantile normalization and subsequent data processing were performed using Applied Biosystems ${ }^{\mathrm{mm}}$ Transcriptome Analysis Console (TAC) Software (Affymetrix, USA). Heat maps representing differentially regulated genes were generated using R software (Vienna University of Economics and Business, Austria). According to the results of microarray, RNAs with fold change $>1.5$ were marked as significantly differentially expressed genes.

\section{Gene annotation and pathway enrichment analysis}

Differentially regulated mRNAs were put into gene ontology (GO) biological process enrichment and Kyoto Encyclopedia of Genes and Genomes (KEGG) signaling pathways analysis as previously depicted [20, 21, 24]. The results of bioinformatics analysis were plotted as bubble chart using $\mathrm{R}$ packaging 'ggplot2' $[25,26]$. The interaction relationship between these mRNAs were evaluated by applying Search Tool for the Retrieval of Interacting Genes (STRING) which is an online tool designed to evaluate the protein-protein interaction (PPI) 
information [27]. Node connectivity (degree) were calculated using contextual hub analysis tool in Cytoscape software to identify hub genes [28, 29]. Nodes with degree $>10$ were considered as highly connected nodes (hub genes).

\section{In vivo nude mice xenograft model}

As we previously depicted, $2 \times 10^{6}$ ECA109 or KYSE410 cells were inoculated subcutaneously to the hind limb of $\mathrm{BALB} / \mathrm{c}$ nude mice (CAMS) to construct the ESCC xenograft model. Animals injected with ECA109 or KYSE410 cells were randomly divided into five groups. When the nude mice generate tumors with a size of 100 $\mathrm{mm}^{3}$, PBS, $10 \mu \mathrm{g}$ exosomes from normal squamous esophageal epithelial cell line (HET-1A), exosome release inhibitor (GW4869, $1 \mathrm{mg} / \mathrm{kg}$ ), $10 \mu \mathrm{g}$ exosomes from normoxic ECA109 or KYSE410 cells (norm-Exo), or $10 \mu \mathrm{g}$ exosomes from hypoxic ECA109 or KYSE410 cells (hypo-Exo) were then injected into the center of tumor sites every 2 days. The tumor size was measured every 5 days. After 40 days, the nude mice were sacrificed and tumors were taken out to measure the weight. The proliferation and angiogenesis status of tumor tissues and lung metastasis were determined using histological examination. Tumor volume was calculated with the formula: length $\times$ width $^{2} \times 0.5[22,30]$. Three mice were used for each group. One representative of three independent experiments was shown.

\section{Immunohistochemistry}

Primary tumor tissues and lungs were firstly fixed in formalin and embedded in paraffin, and then cutted into sections. The lung sections were then stained by hematoxylin and eosin. For immunohistochemistry staining, sections were incubated with primary antibodies (Ki-67, CD31) (Servicbio, China) at $4{ }^{\circ} \mathrm{C}$ overnight, followed by secondary antibody. The stained tumor sections were visualized using the Zeiss inverted microscope (Zeiss, Germany). The number of positive staining tumor cells were counted in three random microscopic fields.

\section{Statistical analysis}

Statistical analysis was conducted using GraphPad Prism 6 software. Unpaired t-test was used to analyze the differences between two groups. Comparisons among more than two groups were performed using one-way ANOVA followed by Holm-Sidak's multiple comparison tests. $P$ value $<0.05$ was considered significant. All data are expressed as mean \pm standard deviation (SD).

\section{Results}

Characterization of exosomes from ESCC cells

The morphology of purified extracellular vesicles from the supernatant of ESCC cells (ECA109, KYSE410) cultured under normoxic conditions was visualized by transmission electron microscopy (Fig. 1a). NTA showed that the particle size distribution of purified extracellular vesicles were between 20 and $200 \mathrm{~nm}$ (Fig. 1b).

Western blotting analysis demonstrated that specific exosome markers (CD9 and TSG101) were enriched in purified extracellular vesicles from the supernatant of both ECA109 and KYSE410 (Fig. 1c). All together, these results confirmed that exosomes were extracted from the ESCC cell supernatant.

\section{ESCC cells derived exosomes were internalized by endothelial cells}

To investigate interactions between exosomes from ESCC cells and HUVECs, exosomes stained with the PKH26 were incubated with HUVECs. The uptake of exosomes in HUVECs was recorded by confocal microscopy at $15 \mathrm{~min}, 60 \mathrm{~min}, 2 \mathrm{~h}$ and $4 \mathrm{~h}$. Then HUVECs were stained with iFluor 488 for Phalloidin and DAPI for nucleus. Figure 2 demonstrated that exosomes uptake by HUVECs started after $15 \mathrm{~min}$ of incubation and increased constantly over time. The internalized exosomes mainly located in the cytoplasm of endothelial cells. In contrast, no labeling was observed in the control group (HUVECs cultured without exosomes) (Exosome (-)). Our results here suggested the internalization of exosomes, from both ECA109 and KYSE410, by HUVECs.

\section{Hypoxic exosomes promoted endothelial cell proliferation, cell cycle progression and migration}

Exosomes were isolated from ESCC cells which cultured in hypoxic and normoxic environment respectively. Then we investigated the effects of normoxic exosomes (norm-Exo) and hypoxic exosomes (hypo-Exo) on HUVECs proliferation, migration and invasion. In the proliferation analysis, norm-Exo increased the colony formation number of HUVECs. Moreover, hypo-Exo played a better role in boosting the colony formation number than norm-Exo or control group (Fig. 3a-b). Follow-up cell cycle analysis demonstrated that normExo promoted HUVECs proliferation by facilitating the cell cycle progression, leading to an increased $S$ and G2/M population. Besides, hypo-Exo played a better role in facilitating the cell cycle progression in HUVECs. (Figure 3c-d). In transwell assay, norm-Exo enhanced the migration and invasion of HUVECs. Similar to the results of cell cycle, HUVECs cultured with hypo-Exo exhibited higher motility than those 

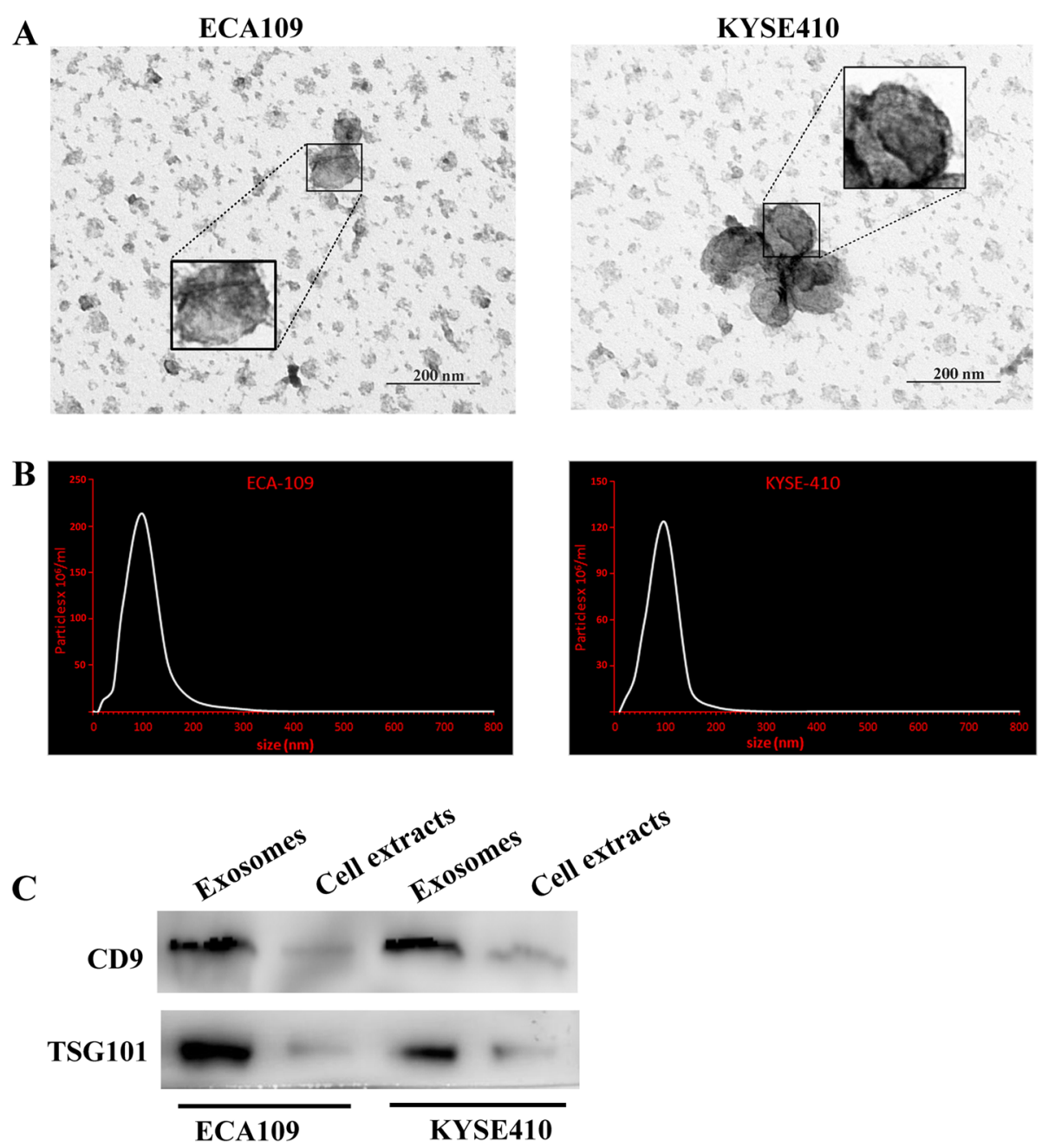

Fig. 1 Identification of the purified extracellular vesicles. a Transmission electron micrographs of extracellular vesicles derived from ECA109 and KYSE410. $\mathbf{b}$ The nanoparticle concentration and size distribution of the extracellular vesicles derived from ECA109 and KYSE410. c The expression level of CD9 and TSG101 (exosome specific markers) in extracellular vesicles

cultured with norm-Exo (Fig. 3e-h). These results suggest that hypoxic ESCC cell-derived exosomes promoted endothelial cell proliferation, migration and invasion in vitro.

\section{Hypoxic exosomes accelerated vascular formation in vitro} and angiogenesis in vivo

In order to determine the effects of ESCC cell-derived exosomes on vascular formation ability of HUVECs, we performed the tube formation assay based on matrigel matrix. Firstly, we observed that both norm-Exo and hypo-Exo significantly increased the number of nodes and total tube length in network structures compared with those cultured without exosomes (Exosome $(-)$ ), indicating the pro-angiogenesis ability of exosomes from ESCC cells in vitro. Further analysis revealed that HUVECs cultured with hypo-Exo exhibited better vascular formation ability compared with those cultured with norm-Exo (Fig. 4a-b).

The angiogenic potential of exosomes from ESCC cells was further evaluated in vivo by examining the recruitment of vasculature into subcutaneously implanted Matrigel plugs containing exosomes. Figure $4 \mathrm{c}$ showed that the plugs containing norm-Exo or hypo-Exo became more vascularized than implants without exosomes (Exosome (-)).

Histological examination showed that matrigel plug containing norm-Exo or hypo-Exo showed much more micro-vessels. The vascular density of Matrigel plugs containing hypo-Exo were significantly higher than those containing norm-Exo. (Figure 4d-e) These data suggested that hypo-Exo played a better role for endothelial cells recruitment and vascular organization than normExo both in vitro and in vivo. 


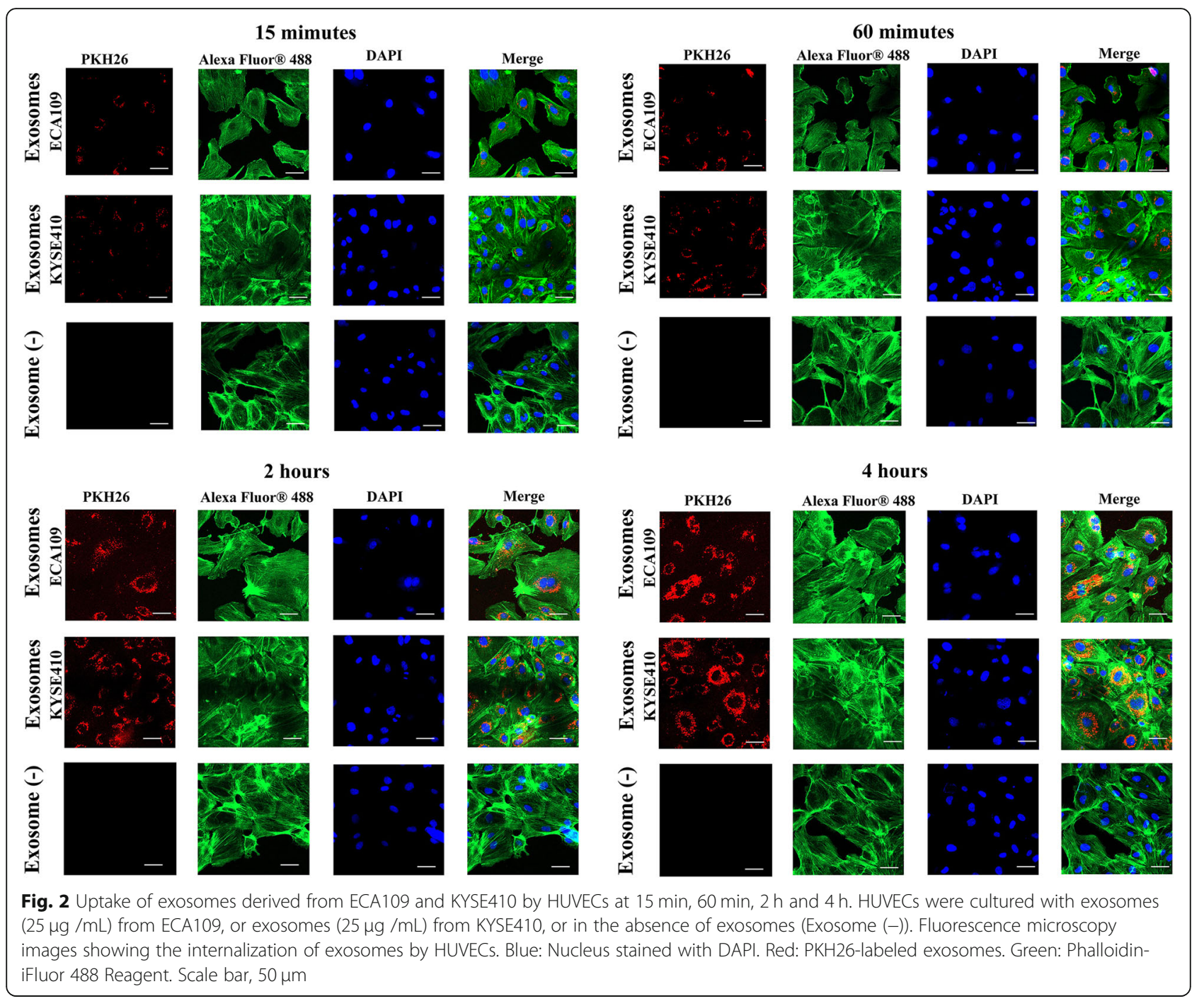

Hypoxic exosomes facilitated ESCC tumor growth, enhanced angiogenesis and promoted metastasis To further determine the angiogenic role of exosomes in vivo, an ESCC tumor-bearing nude mice model was established and treated with exosomes from HET-1A, exosome release inhibitor (GW4869), normoxic ESCCsecreted exosomes (norm-Exo) or hypoxic ESCC-secreted exosomes (hypo-Exo). In mice xenograft model, norm-Exo injection promoted the tumor proliferation and resulted in an increased tumor size. Moreover, administration of hypo-Exo substantially accelerated tumor expansion at later time points, resulting in a significant increase in final tumor volume and tumor weight compared with norm-Exo and control group. (Fig. 5a-f). In support of these data, tumors with hypo-Exo showed substantially enhanced tumor cell proliferation compared with norm-Exo and untreated controls with more tumor cells positively stained with Ki67 (Fig. 5g-j). CD31 immunohistochemical analysis suggested that ESCC tumors grown in the presence of hypo-Exo exhibited enhanced vascularization and significantly greater microvessel density, compared with tumors in norm-Exo and control group (Fig. 5k-n). Hence, although norm-Exo showed a tendency to stimulate tumor growth, hypo-Exo exosomes were significantly more potent.

Also, a significant larger number of lung metastasis nodules was observed in hypo-Exo group than normExo and control group. Immunohistochemistry stain of the lungs showed that the metastasis nodules in hypoExo group were more extensive which was consistent with the general observation results (Fig. 5o-r).

Besides, exosomes from normal squamous esophageal epithelial cell line (HET-1A) had no significant effects on ESCC progression. However, exosome release inhibitor, GW4869, suppressed ESCC tumor proliferation or angiogenesis in situ or distant lung metastasis notably in both ECA109 and KYSE410 mice xenograft model. 


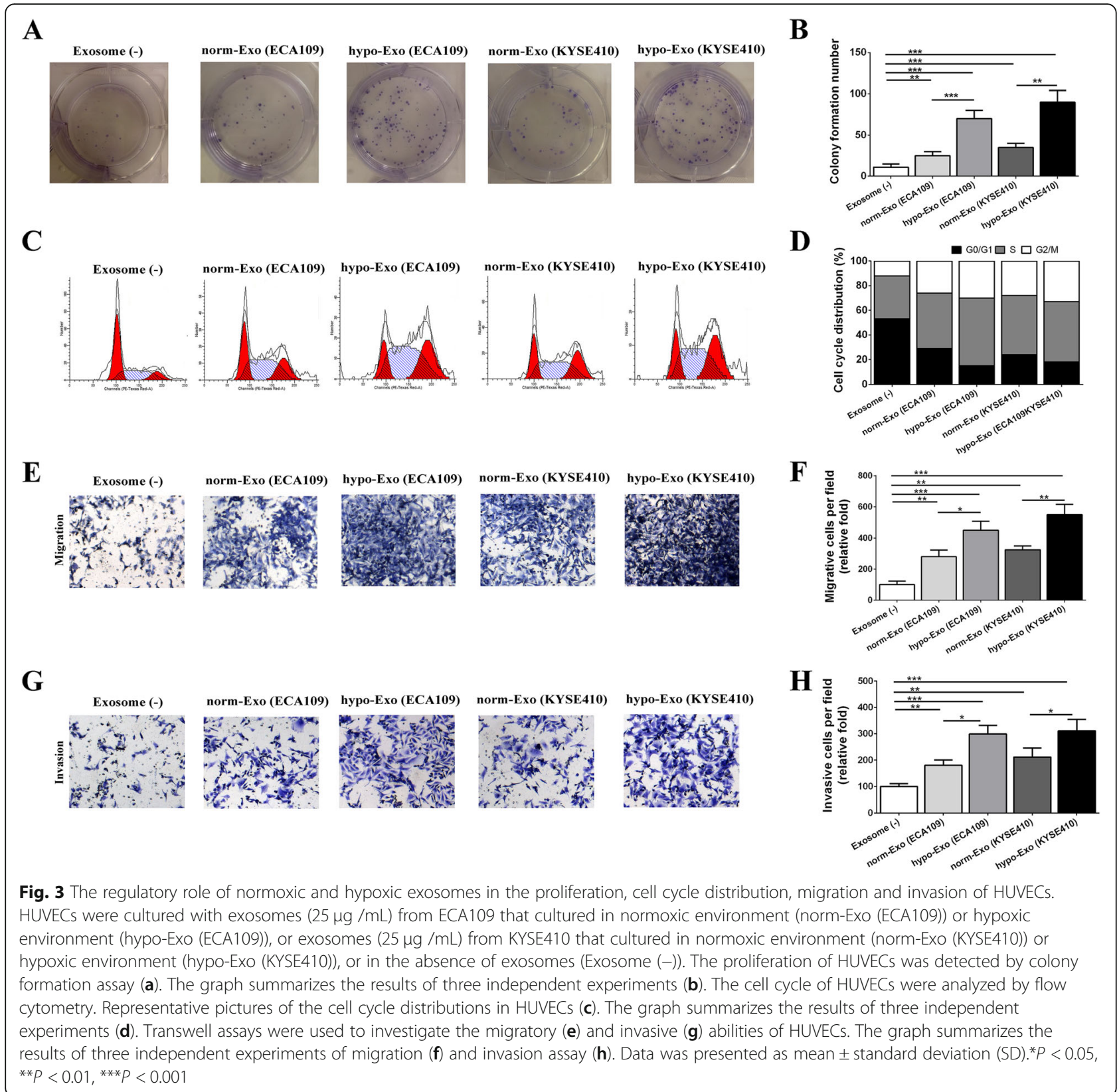

\section{Microarray analysis of transcriptome in HUVECs treated with hypoxic and normoxic exosomes}

To elucidate the functional mechanism and angiogenic effects of ESCC exosomes in endothelial cells, we compared the transcriptome in HUVECs which uptake the hypo-Exo or norm-Exo using microarray analysis. The experiment composed of three groups: HUVECs incubated without exosomes (control group), HUVECs incubated with exosomes form KYSE410 that cultured in normoxic condition (norm-Exo group) and HUVECs incubated with exosomes from KYSE410 that cultured in hypoxic condition (hypo-Exo group). By comparing the transcriptome of HUVECs in norm-Exo and control group, a total of 1656 mRNAs, 1002 lncRNAs and 1312 circular RNAs were dysregulated in HUVECs after the internalization of normoxic ESCC exosomes (Fig. 6a). On the other side, a total of 2171 mRNAs, 1533 lncRNAs and 1698 circular RNAs were dysregulated in HUVECs after the internalization of hypoxic ESCC exosomes (Fig. 6b) (Additional files 1 and 2).

Results aforementioned showed that hypoxia facilitate the angiogenic effects of exosomes, hence, we focused on the RNAs which experienced an up-or down-regulation in both norm-Exo group and hypo-Exo group. According to the intersection of transcriptome, we identified 839 mRNAs which down-regulated in both 


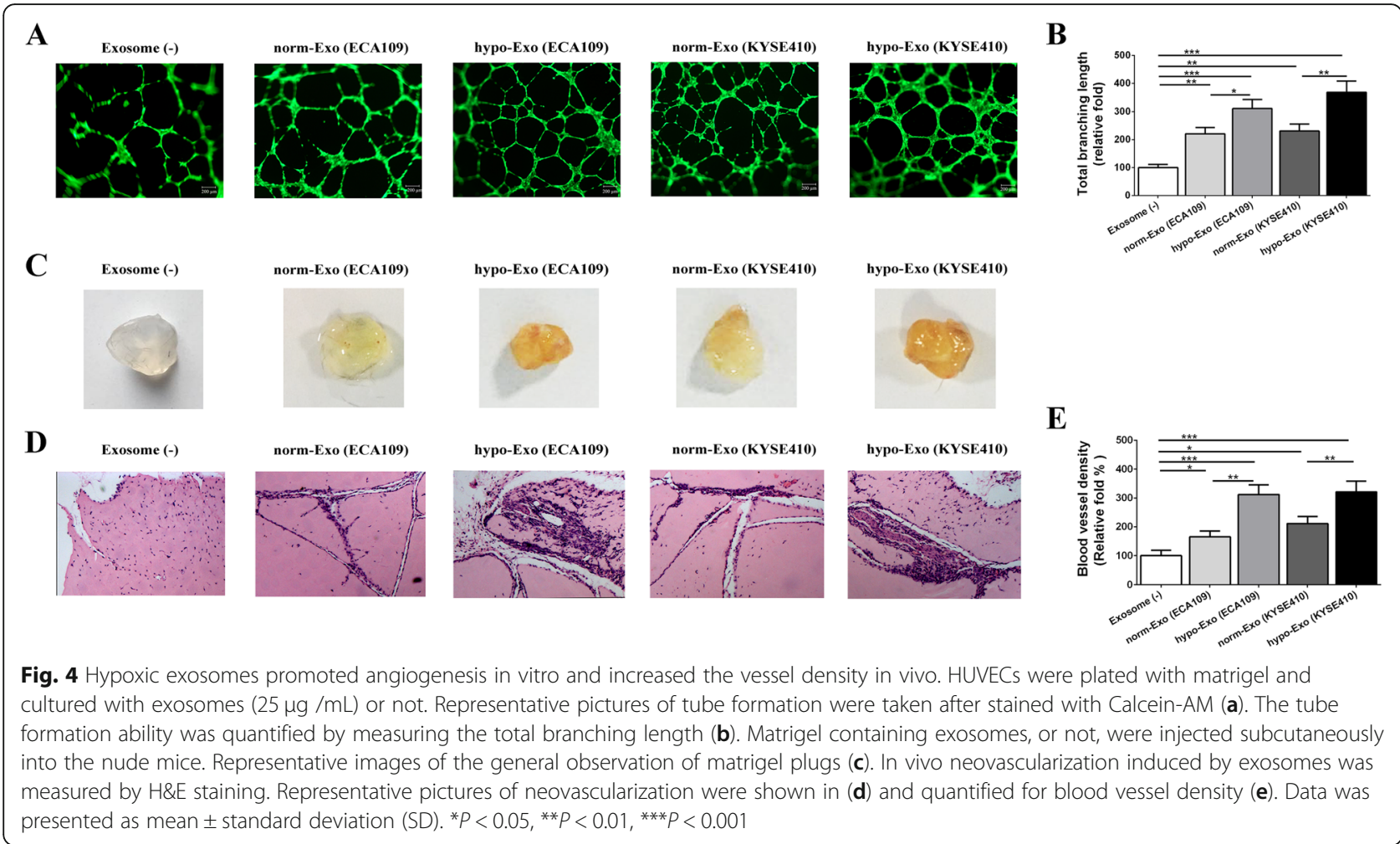

norm-Exo and hypo-Exp groups (Fig. 6c). In this way, 113 up-regulated mRNAs (Fig. 6d), 232 down-regulated lncRNAs (Fig. 6e), 99 up-regulated lncRNAs (Fig. 6f), 692 down-regulated circular RNAs (Fig. 6g) and 86 upregulated circular RNAs (Fig. 6h) were identified in both norm-Exo and hypo-Exp groups.

\section{Function annotation and signaling pathways analysis of differentially expressed genes}

Subsequently, we further explored the potential functions of differentially expressed genes in both norm-Exo group and hypo-Exo group, we put the dysregulated mRNAs, including 839 down-regulated mRNAs and 113 up-regulated mRNAs into gene ontology (GO) biological process enrichment and Kyoto Encyclopedia of Genes and Genomes (KEGG) signaling pathways analysis. According to Fig. 7a, these genes were significantly enriched in cancer-related GO terms, such as cell proliferation (GO: 0008283), cell cycle (GO: 0007049), migration (GO: 0016477) and angiogenesis (GO: 0001525). Moreover, KEGG signaling pathways analysis showed that dysregulated mRNAs also play a role in cancer-related pathways such as cell cycle (has: 04110) and NODlike receptor signaling pathway (hsa:04621) (Fig. 7b). Then we plotted the PPI network of dysregulated mRNAs enriched in cell cycle (GO: 0007049) (Fig. 7c) and cell migration (GO: 0016477) (Fig. 7d). Through calculating the connectivity degree of genes in the cell cycle network, we identified the hub genes PLK1, BUB1 and AURKA which were considered highly correlated with other genes in the network and played critical roles in modulating cell cycle (Fig. 7c). Similarly, VEGFA, CXCL8 and CCL2 were hub genes in in the cell migration network (Fig. 7d).

\section{Discussion}

Hypoxia, or low oxygen tension, has emerged as a specific and general feature of the tumor microenvironment which contribute to cancer development and aggressiveness [19]. In oncology, it still remains to be elucidated that how cancer cells adapt to the hypoxia environment. Actually, hypoxic microenvironment induces a series of adaptive mechanisms including phenotypic modulation of stromal cells in the tumor microenvironment that can prolong the survival and mediate the dissemination of malignant cells [31, 32]. Importantly, hypoxia activates the angiogenic signaling pathway and results in the sprouting of blood vessels from the surrounding tissues into the tumor, during which the intercellular communication between cancer cells and endothelial cells is indispensable [33].

Exosomes are small vesicles of endocytic origin released by most cell types. Exosomes can facilitate eukaryotic intercellular communication under a wide range of normal physiological contexts. In malignancies, this regulatory circuit is co-opted to promote cancer cell survival and outgrowth [34]. 
A

\begin{tabular}{|c|c|c|}
\hline PBS & $\begin{array}{c}\text { Exosomes } \\
\text { (HET-1A) }\end{array}$ & GW4869 \\
\hline
\end{tabular}

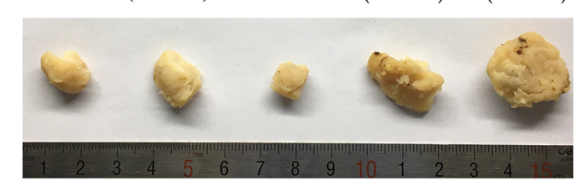

C

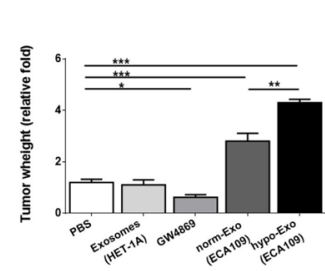

D

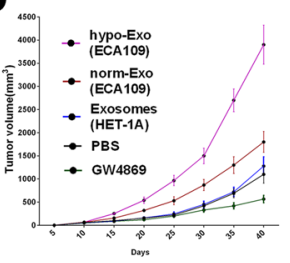

G

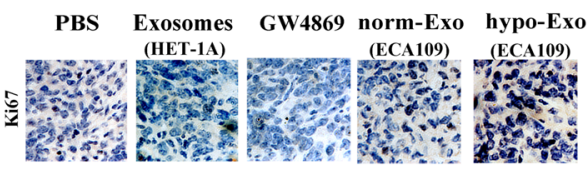

I

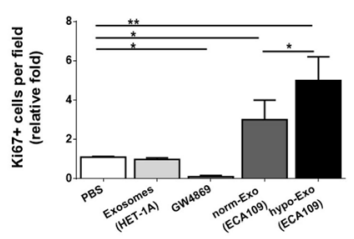

$\mathbf{K}$

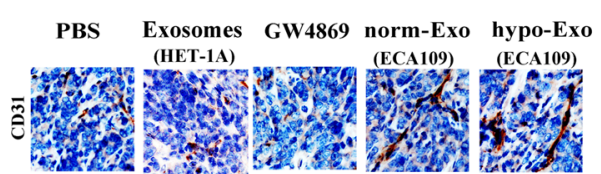

M

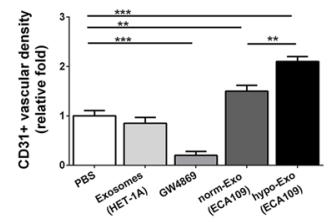

$\mathbf{O}$

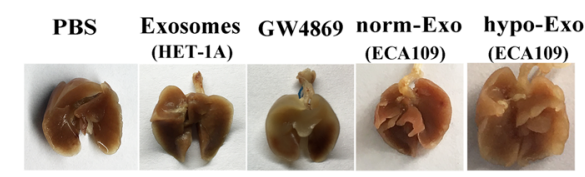

$\mathbf{Q}$
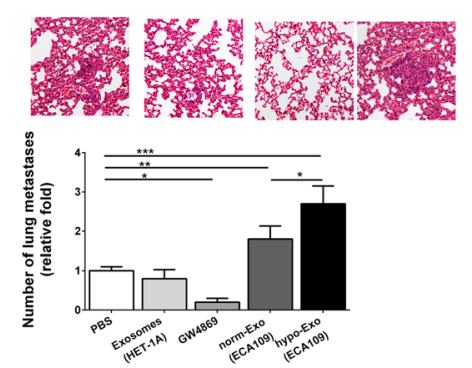

B

KYSE410

$\begin{array}{ccccc}\text { PBS } & \begin{array}{c}\text { Exosomes } \\ \text { (HET-1A) }\end{array} & \text { GW4869 } & \begin{array}{c}\text { norm-Exo } \\ \text { (KYSE410) }\end{array} & \begin{array}{c}\text { hypo-Exo } \\ \text { (KYSE410) }\end{array}\end{array}$

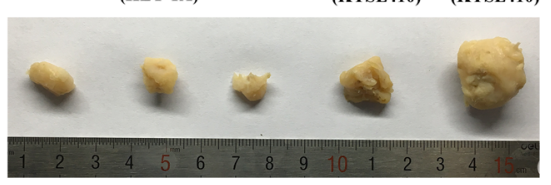

$\mathbf{E}$

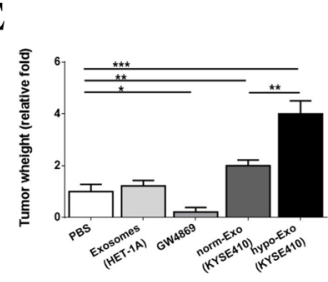

F

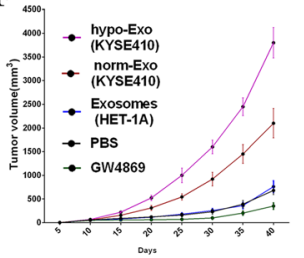

H PBS Exosomes GW4869 norm-Exo hypo-Exo

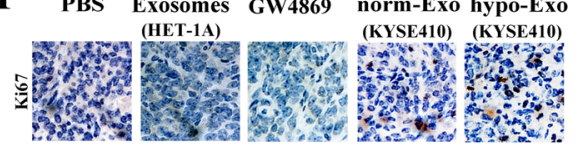

J

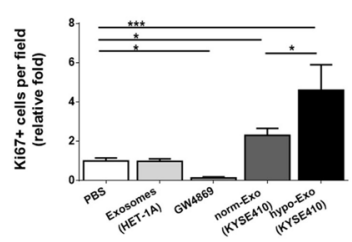

L PBS Exosomes GW4869 norm-Exo hypo-Exo

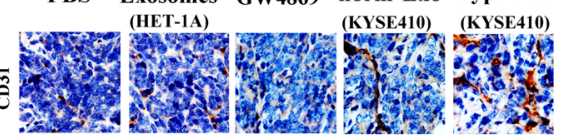

$\mathbf{N}$

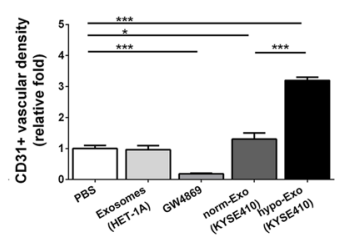

$\mathbf{P}$

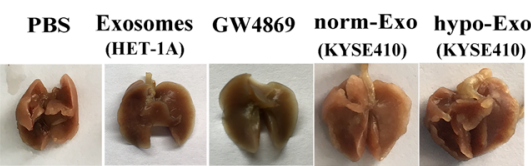

$\mathbf{R}$
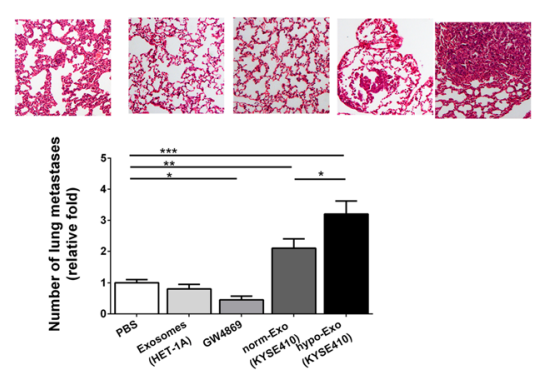

Fig. 5 (See legend on next page.) 
(See figure on previous page.)

Fig. 5 Hypoxic exosomes promoted tumor proliferation and angiogenesis in situ and facilitated lung metastasis. Xenograft transplanted tumor models were established through subcutaneously injecting ECA109 (a) or KYSE410 (b) into the nude mice. Then animals were randomly divided into five groups and injected with PBS, $10 \mu \mathrm{g}$ exosomes from normal squamous esophageal epithelial cell line (HET-1A), exosome release inhibitor (GW4869, 1 mg/kg), $10 \mu \mathrm{g}$ exosomes from normoxic ECA109 or KYSE410 cells (norm-Exo), or $10 \mu \mathrm{g}$ exosomes from hypoxic ECA109 or KYSE410 cells (hypo-Exo) every 2 days (a and b). ECA109 or KYSE410 tumor weigh was measured and shown in $\mathbf{c}$ and $\mathbf{e}$. Tumor growth curves for ECA109 or KYSE410 tumor models were shown in $\mathbf{d}$ and $\mathbf{f}$. Then tumors were analyzed by immunofluorescence microscopy for Ki67 and CD31.

Representative pictures of Ki67 were shown in $\mathbf{g}$ and $\mathbf{h}$, and quantified for cell proliferation (i and $\mathbf{j}$ ). Representative pictures of CD31 were shown in $\mathbf{k}$ and $\mathbf{I}$, and quantified for vascular density ( $\mathbf{m}$ and $\mathbf{n}$ ). Representative images of the general observation the lungs with metastasis nodules and the corresponding H\&E images of the tumor edges in lungs (o and $\mathbf{p}$ ). The metastasis lung nodules were quantified (q and $\mathbf{r}$ ). Data was presented as mean \pm standard deviation (SD). ${ }^{*} P<0.05,{ }^{*} P<0.01,{ }^{* *} P<0.001$

Here, we provide evidence that exosomes from ESCC cells constitute a potent mediator of intercellular communication between cancer and vascular endothelial cells. We firstly characterized the exosomes from ESCC cells and visualized the internalization of these exosomes by HUVECs. Subsequently, we verified that exosomes derived from ESCC cells enhanced HUVECs proliferation through regulating its cell cycle. The invasive ability of HUVECs was also activated by exosomes from ESCC. In vitro and in vivo assay showed that exosomes from ESCC cells significantly promoted the formation of capillary-like structures of HUVECs and improved the microvessel density in transplanted gel plugs from nude mice. Based on these results, we concluded that exosomes from ESCC cells enhanced angiogenesis in TEM.

Moreover, previous studies have demonstrated that hypoxia promotes the release of exosomes by cancer cells and the secreted proteome from hypoxic carcinoma cells are closely associated with exosomes, indicating a potential role of exosomes in regulating the hypoxic response of tumor cells $[35,36]$. In the present study, we compared the biological modulatory role of exosomes from ESCC cells cultured in normoxic and hypoxic condition by performing a series function experiments aforementioned. Hypoxia augmented the angiogenic effects of exosomes derived from ESCC and resulted in the enhancement of vascular formation. These results suggested that exosomes might act as a potential regulator which participate in the hypoxia-driven, phenotypic alteration of endothelial cells in TEM and, as a result, affect angiogenesis.

It has been widely accepted that angiogenesis is closely related with tumor growth metastasis [37]. Hence, we further explored the contribution of hypoxic exosomes in ESCC angiogenesis, tumor growth and metastasis. In tumor-bearing mice model, hypoxic exosomes significantly enhanced the ESCC progression by promoting the proliferation of cancer cells, vascular formation and metastasis. Based on the results we mentioned above, we supposed that hypoxia altered the content of exosome secreted by ESCC and enhanced the vascular formation by endothelial cells after internalized these exosomes in
TEM. The newly-formed vascular alleviated the hypoxia condition in TEM of ESCC and in turn contribute to the tumor growth. Moreover, increased micro vessel density in TEM facilitated the escape of cancer cells into the bloodstream. Disseminated tumor cells in the blood paved the way for follow-up establishment of metastatic colonies in secondary sites.

On the other side, tumor-derived exosomes are demonstrated to be the major drivers of the pre-metastatic niche. Previous studies demonstrated that exosomes destroyed endothelial barriers and increased vascular permeability which provide an escape route for the tumor cells to enter the circulation [38]. Besides, in the target organ, a microenvironment suitable for tumor metastasis has been created by exosomes for tumor metastasis before cancer cells transfection [39, 40]. Hence, whether hypoxia take an effect on the cancer cells secreted exosomes which change the vascular permeability and result in the pre-metastatic niche formation in ESCC deserves further study.

Furthermore, exosomes carry genetic messages, such as mRNA, miRNA, IncRNA and circular RNA, and can be internalized by the recipient cells [10]. The mRNAs presented in exosomes has been demonstrated to be functional and translatable, which means they are capable of encoding polypeptides and supporting protein synthesis in host cells. Specific protein production may provide the necessary signal(s) to modulate the function of the recipient cells $[41,42]$. The regulatory capacity of noncoding RNAs, such as lncRNA and miRNA, in the exosomes have also been found as extensive. Noncoding RNAs in the exosomes may act as ceRNAs and interfere with mRNAs [43]. Hence, in order to explore the mechanisms underlying the phenotypic modulation effects of exosomes in angiogenesis, we performed the comprehensive analyses of the transcriptome in HUVECs after the internalization of hypoxic and normoxic ESCC exosomes.

As we have mentioned above, hypoxia facilitate the angiogenic effects of exosomes, we focused on the RNAs which experienced an up-or down regulation in both of the two groups. We applied gene annotation and 


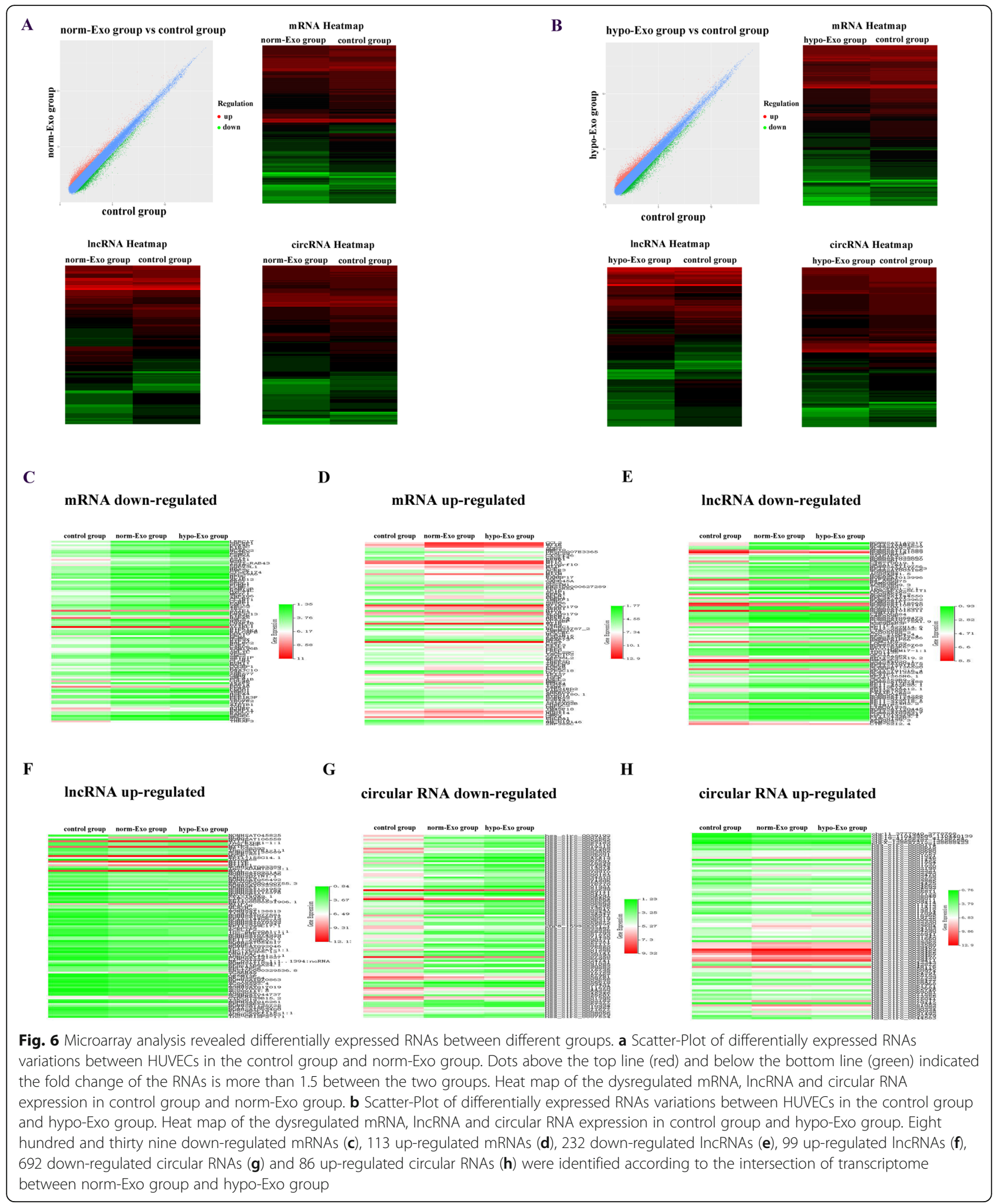

pathway enrichment analysis on the mRNAs to identify their functions. Results showed that these mRNAs were significantly enriched in biological processes such as cell proliferation and migration, and pathways such as cell cycle. Hub genes were also identified which were considered as topologically important to the structure of the network and played crucial roles in the biological process. In cell cycle term (GO: 0007049), PLK1, BUB1 


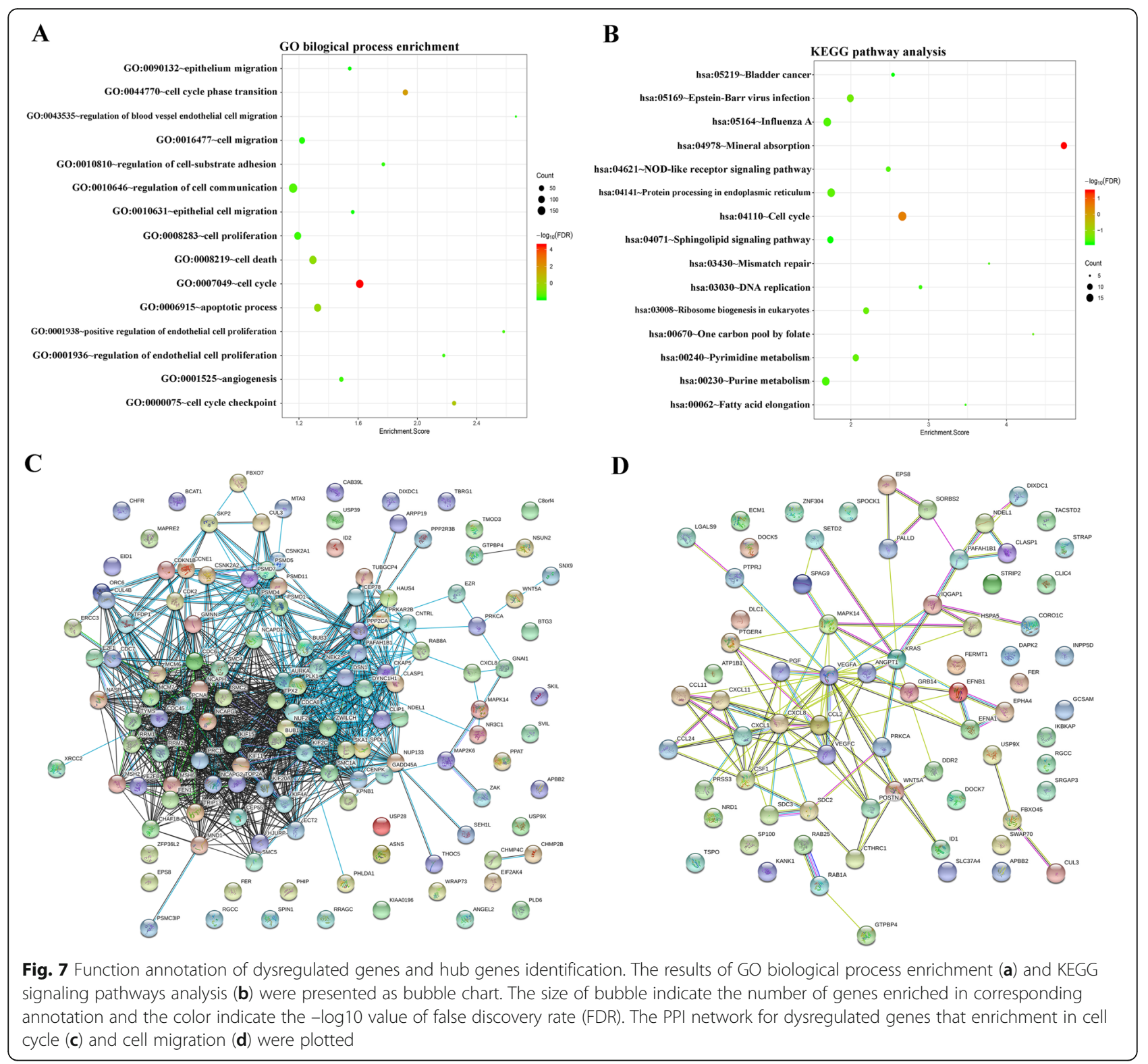

and AURKA were identified as hub genes in cell cycle regulation. Previous studies demonstrate that PLK1-mediate the activation of phosphorylates glucose-6-phosphate dehydrogenase which is critical for the promoting the cell cycle progression and tumor growth in liver cancer and cervical cancer [44]. Moreover, BUB1-PLK1 complex mediate the phosphorylation of $\mathrm{Cdc} 20$ and inhibit the anaphase-promoting complex or cyclosome $(\mathrm{APC} / \mathrm{C})$ which result in the promotion of spindle checkpoint signaling in cervical cancer and osteosarcoma [45]. Silence of AURKA inhibits tumor growth by inducing apoptosis and G2/M cell cycle arrest in human osteosarcoma and breast cancer $[46,47]$.

VEGFA, CXCL8 and CCL2 were identified as the hub genes in cell migration process (GO: 0016477). It is generally accepted that VEGFA play key roles in angiogenesis [48]. The epithelial-mesenchymal transition effect of VEGFA is also verified in cancer and retinal pigment epithelial cells $[49,50]$. CCL2 and CXCL8 induces epithelial-mesenchymal transition in colon cancer and bladder cancer [51, 52]. In view of this, we hypothesized that hypoxia might alter the transcriptome of exosomes from cancer cells and mediated the dysregulation of effector molecules, which triggered a series of cascade reaction, in host cells-HUVECs after internalization. Hence, we next planned to identify the hub genes which induced the angiogenesis-related signaling pathway activation and resulted in phenotype alteration of endothelial cells in the hypoxic TEM of ESCC. 


\section{Conclusions}

In summary, our data suggests that exosomes in ESCC represents a potentially targetable regulating factor in the hypoxia-driven tumor development. This study sheds light on the possible application of exosomes to fight against cancer progression and metastasis.

\section{Additional files}

Additional file 1: Differentially expressed RNA between control group and hypo-Exo group. (XLSX 2643 kb)

Additional file 2: Differentially expressed RNA between control group and norm-Exo group. (XLSX $1939 \mathrm{~kb})$

\section{Abbreviations}

ESCC: Esophageal squamous cell carcinoma; FBS: Fetal bovine serum; GO: Gene ontology; HUVECs: Human umbilical vein endothelial cells; KEGG: Kyoto Encyclopedia of Genes and Genomes; LncRNA: Long noncoding RNA; PPI: Protein-protein interaction; STRING: Search Tool for the Retrieval of Interacting Genes; TEM: Tumor microenvironment

\section{Acknowledgements}

Not applicable.

\section{Authors' contributions}

$Y M$ and ZF contributed to the study design, experiment performance and drafting the article. LD, YZ, YZ, CW, QZ, SY, LC, XZ and XL contributed to the experiment performance and prepared the figures. $Y W$ contributed to the experiment performance and revision of the manuscript. All authors read and approved the final manuscript.

\section{Funding}

This work was supported by Scientific Research Fund of Hebei Health and Family Planning Commission (20181192).

\section{Availability of data and materials}

The datasets generated during the current study are available in the Additional files 1 and 2.

\section{Ethics approval and consent to participate}

The Medical Ethics Committees of First Hospital of Qinhuangdao approved this study.

\section{Consent for publication}

Not applicable.

\section{Competing interests}

The authors declare that they have no competing interests.

\section{Author details}

'Department of Oncology, First Hospital of Qinhuangdao, Wenhua Road No. 258, Haigang District, Qinhuangdao 066000, Hebei, China. ²Department of General Surgery, First Hospital of Qinhuangdao, Hebei Medical University, Qinhuangdao, China. ${ }^{3}$ Department of Thoracic Surgery, First Hospital of Qinhuangdao, Qinhuangdao, China.

Received: 22 March 2019 Accepted: 15 August 2019 Published online: 05 September 2019

\section{References}

1. Siegel RL, Miller KD, Jemal A. Cancer statistics, 2017. CA Cancer J Clin. 2017; 67(1):7-30.

2. Allen DC. Oesophageal carcinoma. Lancet. 2013;381(9864):400-12

3. Predescu D, Gheorghe M, Boeriu M, Constantin A, losif C, Anghel R, et al. Molecular factors and criteria for predicting the response to neoadjuvant treatment in patients with esophageal squamous cell carcinoma (ESCC) -- responder/non-responder. Chirurgia (Bucharest, Romania : 1990). 2012; 107(5):583-90.

4. Bielenberg DR, Zetter BR. The contribution of angiogenesis to the process of metastasis. Cancer J. 2015;21(4):267.

5. Viallard C, Larrivee B. Tumor angiogenesis and vascular normalization: alternative therapeutic targets. Angiogenesis. 2017;20(4):409-26.

6. Rey S, Schito L, Wouters BG, Eliasof S, Kerbel RS. Targeting hypoxia-inducible factors for antiangiogenic Cancer therapy. Trends Cancer. 2017;3(7):529-41.

7. De Palma M, Biziato D, Petrova TV. Microenvironmental regulation of tumour angiogenesis. Nat Rev Cancer. 2017;17(8):457-74.

8. Chung AS, Ferrara N. Developmental and pathological angiogenesis. Annu Rev Cell Dev Biol. 2011;27:563-84.

9. Milane L, Singh A, Mattheolabakis G, Suresh M, Amiji MM. Exosome mediated communication within the tumor microenvironment. J Control Release. 2015:219:278-94.

10. Valadi H, Ekström K, Bossios A, Sjöstrand M, Lee JJ, Lötvall JO. Exosomemediated transfer of mRNAs and microRNAs is a novel mechanism of genetic exchange between cells. Nat Cell Biol. 2007;9(6):654.

11. Lowry MC, Gallagher WM, O'Driscoll L. The role of exosomes in breast cancer. Clin Chem. 2015;61(12):1457-65.

12. Kang M, Ren M, Li Y, Fu Y, Deng M, Li C. Exosome-mediated transfer of IncRNA PART1 induces gefitinib resistance in esophageal squamous cell carcinoma via functioning as a competing endogenous RNA. J Exp Clin Cancer Res. 2018;37(1):171

13. Mao L, Li X, Gong S, Yuan H, Jiang Y, Huang W, et al. Serum exosomes contain ECRG4 mRNA that suppresses tumor growth via inhibition of genes involved in inflammation, cell proliferation, and angiogenesis. Cancer Gene Ther. 2018;25(9-10):248-59.

14. Ruivo CF, Adem B, Silva M, Melo SA. The biology of Cancer exosomes: insights and new perspectives. Cancer Res. 2017;77(23):6480-8.

15. Su LL, Chang XJ, Zhou HD, Hou LB, Xue XY. Exosomes in esophageal cancer: a review on tumorigenesis, diagnosis and therapeutic potential. World J Clin Cases. 2019;7(8):908-16.

16. Li W, Zhang L, Guo B, Deng J, Wu S, Li F, et al. Exosomal FMR1-AS1 facilitates maintaining cancer stem-like cell dynamic equilibrium via TLR7/ NFkB/C-Myc signaling in female esophageal carcinoma. Mol Cancer. 2019: 18(1):22.

17. Liao J, Liu R, Shi Y-J, Yin L-H, Pu Y-P. Exosome-shuttling microRNA-21 promotes cell migration and invasion-targeting PDCD4 in esophageal cancer. Int J Oncol. 2016:48(6):2567-79.

18. Mao Y, Wang Y, Dong L, Zhang Q, Wang C, Zhang Y, et al. Circulating exosomes from esophageal squamous cell carcinoma mediate the generation of B10 and PD-1(high) Breg cells. Cancer Sci. 2019. https://doi. org/10.1111/cas.14122. [Epub ahead of print].

19. Kucharzewska P, Christianson HC, Welch JE, Svensson KJ, Fredlund E, Ringner $\mathrm{M}$, et al. Exosomes reflect the hypoxic status of glioma cells and mediate hypoxia-dependent activation of vascular cells during tumor development. Proc Natl Acad Sci U S A. 2013;110(18):7312-7.

20. Mao Y, Fu Z, Zhang Y, Dong L, Zhang Y, Zhang Q, et al. A seven-IncRNA signature predicts overall survival in esophageal squamous cell carcinoma. Sci Rep. 2018;8(1):8823.

21. Mao Y, Fu Z, Zhang Y, Dong L, Zhang $Y$, Zhang Q, et al. A six-microRNA risk score model predicts prognosis in esophageal squamous cell carcinoma. Cell Physiol. 2019;234(5):6810-9.

22. Mao Y, Li L, Liu J, Wang L, Zhou Y. MiR-495 inhibits esophageal squamous cell carcinoma progression by targeting Akt1. Oncotarget. 2016;7(32):51223-36.

23. Malinda KM. In vivo matrigel migration and angiogenesis assay. Methods Mole Biol (Clifton, NJ). 2009;467:287-94.

24. Mao Y, Fu Z, Dong L, Zheng Y, Dong J, Li X. Identification of a 26-IncRNAs risk model for predicting overall survival of cervical squamous cell carcinoma based on integrated bioinformatics analysis. DNA Cell Biol. 2019.

25. Wickham H. ggplot2. Wiley Interdisciplinary Rev Comp Stat. 2015;3(2):180-5.

26. Ginestet C. ggplot2: elegant graphics for data analysis by H. Wickham. J R Stat Soc. 2011:174(1):245-6.

27. Damian S, Andrea F, Stefan W, Kristoffer F, Davide H, Jaime HC, et al. STRING v10: protein-protein interaction networks, integrated over the tree of life. Nucleic Acids Res. 2015;43(Database issue):D447.

28. Muetze T, Goenawan $\mathrm{H}$, Wiencko HL, Bernal-Llinares M, Bryan K, Lynn DJ. Contextual Hub Analysis Tool (CHAT): A Cytoscape app for identifying contextually relevant hubs in biological networks. F1000Research. 2016; 5:1745. 
29. Shannon P, Markiel A, Ozier O, Baliga NS, Wang JT, Ramage D, et al. Cytoscape: a software environment for integrated models of biomolecular interaction networks. Genome Res. 2003;13(11):2498-504.

30. Xue M, Chen W, Xiang A, Wang R, Chen H, Pan J, et al. Hypoxic exosomes facilitate bladder tumor growth and development through transferring long non-coding RNA-UCA1. Mol Cancer. 2017;16(1):143.

31. Pouyssegur J, Dayan F, Mazure NM. Hypoxia signalling in cancer and approaches to enforce tumour regression. Nature. 2006:441(7092):437-43.

32. Finger EC, Giaccia AJ. Hypoxia, inflammation, and the tumor microenvironment in metastatic disease. Cancer Metastasis Rev. 2010;29(2):285-93.

33. Liu Y, Luo F, Wang B, Li H, Xu Y, Liu X, et al. STAT3-regulated exosomal miR-21 promotes angiogenesis and is involved in neoplastic processes of transformed human bronchial epithelial cells. Cancer Lett. 2016;370(1):125-35.

34. Syn N, Wang L, Sethi G, Thiery J-P, Goh B-C. Exosome-mediated metastasis: from epithelial-mesenchymal transition to escape from Immunosurveillance. Trends Pharmacol Sci. 2016;37(7):606-17.

35. King HW, Michael MZ, Gleadle JM. Hypoxic enhancement of exosome release by breast cancer cells. BMC Cancer. 2012;12(1):421.

36. Shao C, Yang F, Miao S, Liu W, Wang C, Shu Y, et al. Role of hypoxiainduced exosomes in tumor biology. Mol Cancer. 2018;17(1):120.

37. Maishi N, Hida K. Tumor endothelial cells accelerate tumor metastasis. Cancer Sci. 2017;108(10):1921-6.

38. Guo Y, Ji X, Liu J, Fan D, Zhou Q, Chen C, et al. Effects of exosomes on premetastatic niche formation in tumors. Mol Cancer. 2019;18(1):39.

39. Gupta GP, Nguyen DX, Chiang AC, Bos PD, Kim JY, Nadal C, et al. Mediators of vascular remodelling co-opted for sequential steps in lung metastasis. Nature. 2007:446(7137):765-70.

40. Pasquier J, Thawadi HA, Ghiabi P, Abu-Kaoud N, Maleki M, Guerrouahen BS, et al. Microparticles mediated cross-talk between tumoral and endothelial cells promote the constitution of a pro-metastatic vascular niche through Arf6 up regulation. Cancer Microenvironment. 2014;7(1-2):41-59.

41. Cao YL, Zhuang T, Xing BH, Li N, Li Q. Exosomal DNMT1 mediates cisplatin resistance in ovarian cancer. Cell Biochem Funct. 2017;35(6):296-303.

42. Shao H, Chung J, Lee K, Balaj L, Min C, Carter BS, et al. Chip-based analysis of exosomal mRNA mediating drug resistance in glioblastoma. Nat Commun. 2015:6:6999.

43. Hsu YL, Hung JY, Chang WA, Lin YS, Pan YC, Tsai PH, et al. Hypoxic lung cancer-secreted exosomal miR-23a increased angiogenesis and vascular permeability by targeting prolyl hydroxylase and tight junction protein ZO1. Oncogene. 2017:36(34):4929.

44. Ma X, Wang L, Huang LY, Yang D, Li T, et al. Polo-like kinase 1 coordinates biosynthesis during cell cycle progression by directly activating pentose phosphate pathway. Nat Commun. 2017:8(1):1506.

45. Jia L, Li B, Yu H. The Bub1-Plk1 kinase complex promotes spindle checkpoint signalling through Cdc20 phosphorylation. Nat Commun. 2016; 7:10818.

46. Jiang Z, Jiang J, Yang H, Ge Z, Wang Q, Zhang L, et al. Silencing of Aurora kinase a by RNA interference inhibits tumor growth in human osteosarcoma cells by inducing apoptosis and G2/M cell cycle arrest. Oncol Rep. 2014;31(3):1249-54.

47. Taylor NJ, Bensen JT, Poole C, Troester MA, Gammon MD, Luo J, et al. Genetic variation in cell cycle regulatory gene AURKA and association with intrinsic breast cancer subtype. Mol Carcinog. 2015;54(12):1668-77.

48. Claesson-Welsh L, Welsh M. VEGFA and tumour angiogenesis. J Intern Med. 2013;273(2):114-27.

49. Zhong Z, Huang M, Lv M, He Y, Duan C, Zhang L, et al. Circular RNA MYLK as a competing endogenous RNA promotes bladder cancer progression through modulating VEGFANEGFR2 signaling pathway. Cancer Lett. 2017; 403:305-17.

50. Du Y, Chen Q, Huang L, Wang S, Yin X, Zhou L, et al. VEGFR2 and VEGF-C suppresses the epithelial-mesenchymal transition via YAP in retinal pigment epithelial cells. Curr Mol Med. 2018;18(5):273-86.

51. Shen $T$, Yang $Z$, Cheng $X, X i a o ~ Y, Y u K$, Cai $X$, et al. CXCL8 induces epithelialmesenchymal transition in colon cancer cells via the PI3K/Akt/NF-kappaB signaling pathway. Oncol Rep. 2017;37(4):2095-100.

52. Wang W, Shen F, Wang C, Lu W, Wei J, Shang A, et al. MiR-1-3p inhibits the proliferation and invasion of bladder cancer cells by suppressing CCL2 expression. Tumour Biol. 2017;39(6):1010428317698383.

\section{Publisher's Note}

Springer Nature remains neutral with regard to jurisdictional claims in published maps and institutional affiliations.
Ready to submit your research? Choose BMC and benefit from:

- fast, convenient online submission

- thorough peer review by experienced researchers in your field

- rapid publication on acceptance

- support for research data, including large and complex data types

- gold Open Access which fosters wider collaboration and increased citations

- maximum visibility for your research: over $100 \mathrm{M}$ website views per year

At BMC, research is always in progress.

Learn more biomedcentral.com/submissions 